\title{
A 23-Year-Old Man with Hyper-IgM Syndrome Presenting with Asymptomatic Violaceous Facial Plaques
}

\author{
Mirelys Barrios $^{a} \quad$ Neera Nathan ${ }^{b, c}$ Ryan Trowbridge ${ }^{b, c}$ \\ Molly Plovanich ${ }^{b, c}$ Rosalynn M. Nazarian ${ }^{d}$ Daniela Kroshinsky ${ }^{b}$ \\ a Department of Radiology, Brigham and Womens Hospital, Boston, MA, USA; ${ }^{b}$ Department of \\ Dermatology, Massachusetts General Hospital, Boston, MA, USA; ${ }^{c}$ Harvard Combined \\ Dermatology Residency Program, Boston, MA, USA; dDepartment of Pathology, \\ Massachusetts General Hospital, Boston, MA, USA
}

\section{Keywords}

Tumid lupus · Hyper-IgM syndrome · Facial plaques - Activation-induced cytidine deaminase deficiency

\section{Abstract}

Tumid lupus is a rare subtype of chronic cutaneous lupus that is characterized by urticaria-like photosensitive plaques. Unlike discoid lupus, it has minimal to no surface change and resolves without scarring. On pathological examination, it may be distinguished from other types of lupus by abundant interstitial mucin deposits. Herein, we describe a case of tumid lupus in a 23-year-old Kuwaiti male with hyper-IgM syndrome. To our best knowledge, this is the first report of tumid lupus in a patient with a primary immunodeficiency.

\section{Case Report}

A 23-year-old Kuwaiti male with hyper-IgM syndrome secondary to autosomal recessive activation-induced cytidine deaminase (AID) deficiency, on regular intravenous immunoglobulin replacement therapy, presented with an 8-month history of an asymptomatic violaceous facial plaque. This lesion started as patches near his nose, then spread to involve his cheeks and to a lesser extent, his forehead. Skin biopsy performed in Kuwait was interpreted 

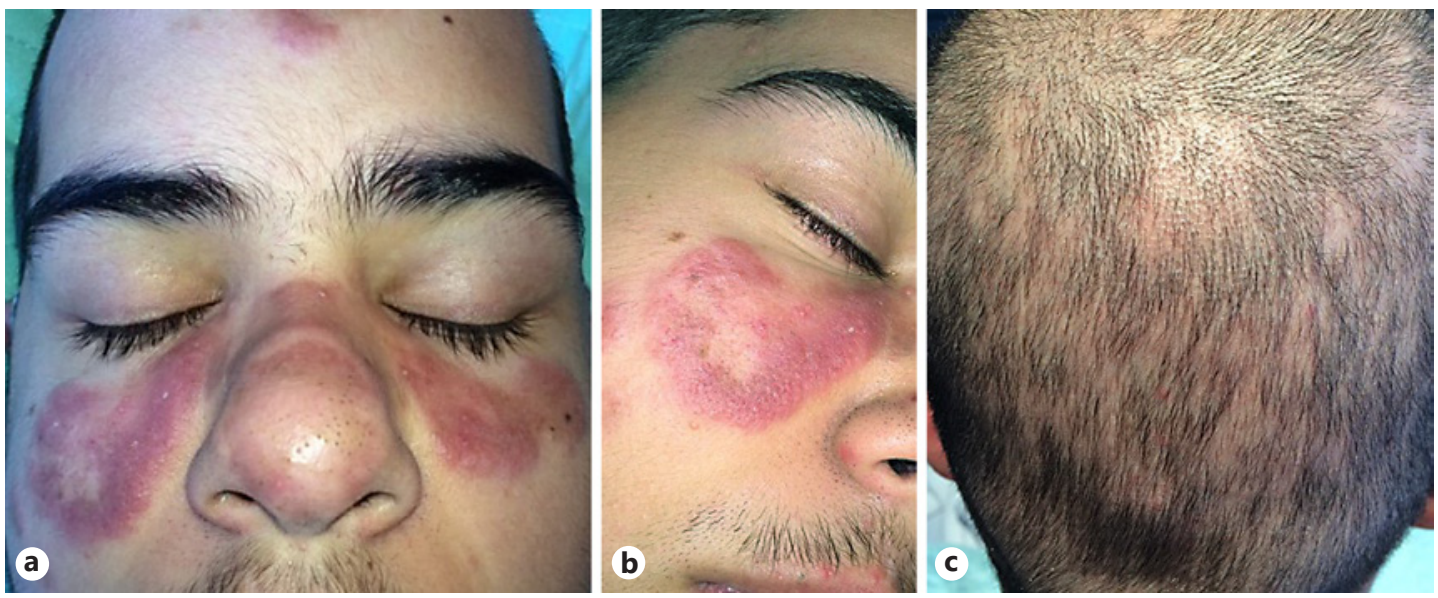

Fig. 1. a, b Red-to-purple, arciform soft plaques with sharp borders on the bilateral malar eminences, nasal bridge, tip and ala, and forehead. c Multifocal erythematous, non-scarring alopetic patches are also present.

as pseudolymphoma, and the patient tried a mid-potency topical steroid and other unknown cream without resolution. He also reported hair thinning and loss over the past year.

Cutaneous examination revealed red-to-purple, arciform soft plaques with sharp borders on the bilateral malar eminences, with less involvement of the nasal bridge, tip and ala, as well as the forehead (Fig. 1a, b). Lesion color did not change with diascopy. Multifocal erythematous, non-scarring alopetic patches were also noted (Fig. 1c).

Initial laboratory workup at presentation was notable for pancytopenia with white blood cell count $3.25 \mathrm{~K} / \mu \mathrm{L}$ (reference range 4.5-11), hemoglobin $11.3 \mathrm{~g} / \mathrm{dL}$ (reference range 13.517.5), and platelets $102 \mathrm{~K} / \mu \mathrm{L}$ (reference range 150-400). Immunologic serologies were consistent with his diagnosis of hyper-IgM syndrome with elevated IgM 3,062 mg/dL (reference range 53-334), low IgA $<7 \mathrm{mg} / \mathrm{dL}$ (reference range 69-309), and normal IgG 1,174 $\mathrm{mg} / \mathrm{dL}$ (reference range 614-1,295). Low complement levels were found with C3 $78 \mathrm{mg} / \mathrm{dL}$ (reference range 81-157) and C4 $10 \mathrm{mg} / \mathrm{dL}$ (reference range 12-39).

A single slide from the previous biopsy in Kuwait was available and re-read as a superficial and deep perivascular and periadnexal lymphohistiocytic infiltrate with plasma cells and dermal edema with a broad histologic differential diagnosis. Treatment with twice-daily clobetasol $0.05 \%$ ointment was begun for suspected pseudolymphoma with some improvement.

\section{Diagnosis and Clinical Course}

A repeat biopsy of the left cheek plaque was performed 1 week after initial presentation, which revealed histological findings similar to previous biopsy, namely superficial and deep perivascular and periadnexal lymphohistiocytic infiltrate with scattered plasma cells and multinucleated giant cells (Fig. 2a). Special stains revealed a CD3+ T-cell infiltrate, basement membrane zone thickening, and abundant interstitial mucin deposition without interface alteration (Fig. 2b, c). Additional stains demonstrated a mixed population of CD4+ and CD8+ $\mathrm{T}$ cells. There was no significant increase in CD34 expression in dermal fibroblasts. Positive immunoreactivity was identified for IgA, IgG, IgG whole, IgM, and C3 along the basement membrane zone ( 2 to $3+$, granular). No microorganisms including spirochetes were identified 

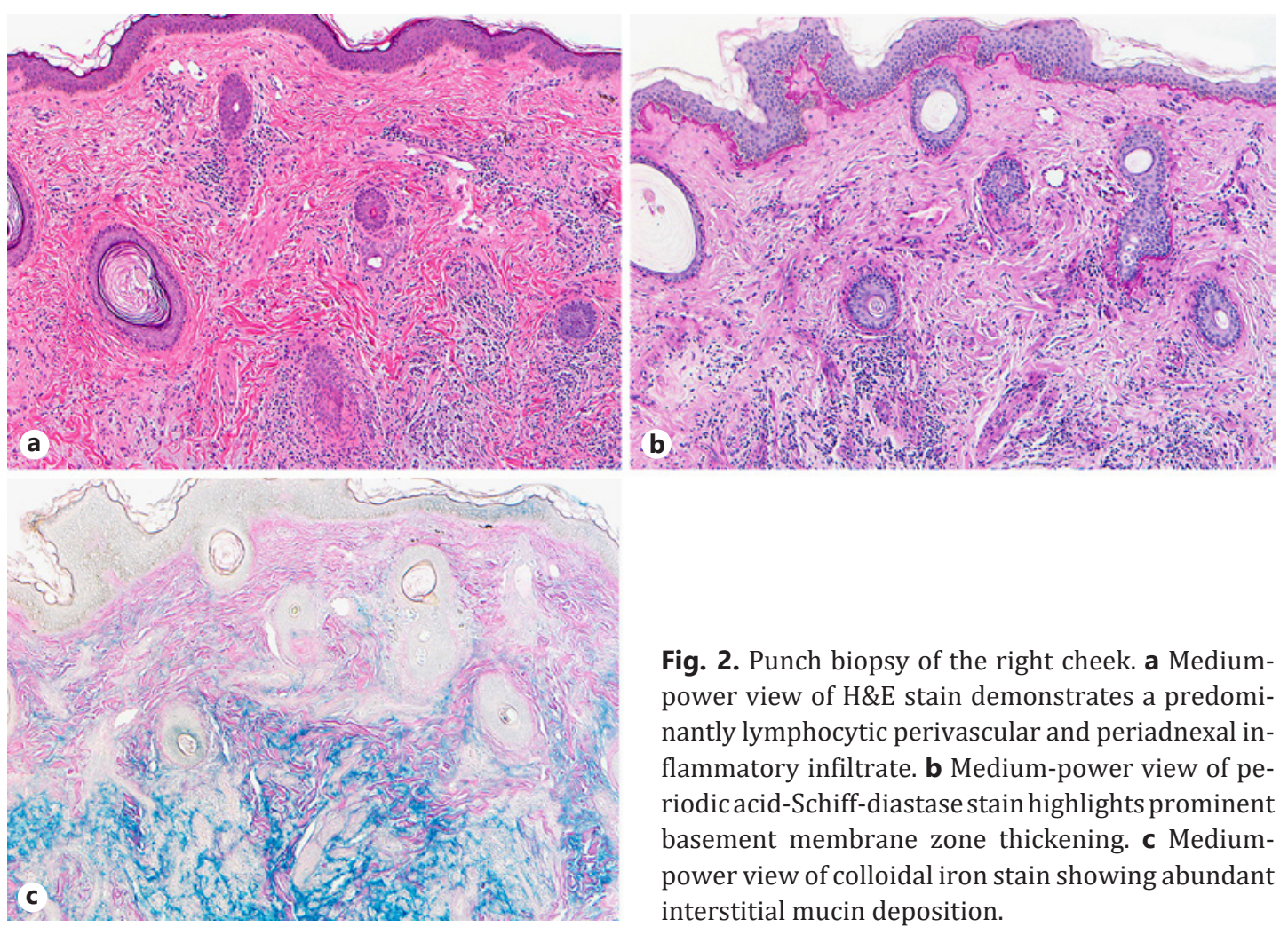

Fig. 2. Punch biopsy of the right cheek. a Mediumpower view of H\&E stain demonstrates a predominantly lymphocytic perivascular and periadnexal inflammatory infiltrate. b Medium-power view of periodic acid-Schiff-diastase stain highlights prominent basement membrane zone thickening. c Mediumpower view of colloidal iron stain showing abundant interstitial mucin deposition.

on AFB, Fite, PAS/D, and Giemsa stains. Elastic stain showed no significant decrease in dermal elastic fibers. No amyloid was identified on a Congo red stain.

Rheumatologic workup revealed positive ANA (1:160) and positive dsDNA (1:40). Antibodies for SS-A (Ro), SS-B(La), and SCL-70 were negative. Infectious disease workup was positive for treponemal antibody and elevated RPR (1:2). The patient was found to be negative for HIV-1/2 Ab/Ag, HBV, HCV, CMV, and Cryptococcus. However, the aforementioned positive autoimmune antibodies and syphilis markers were difficult to interpret in the setting of recent intravenous immunoglobulin infusions to manage his primary immunodeficiency.

Clinical and histopathological examination was most consistent with tumid lupus. The facial plaque improved with treatment with oral prednisone (15 mg daily) and hydroxychloroquine (200 mg twice daily). The patient was offered empiric treatment for syphilis; however, in light of no prior sexual history, he declined treatment with penicillin at the time of hospitalization.

\section{Discussion}

Lupus tumidus erythematous, or tumid lupus, is considered by most to be a subtype of chronic cutaneous lupus [1]. It is characterized by erythematous, succulent plaques with a smooth surface, occasionally in an annular configuration [2]. These lesions are most commonly distributed on the face, torso, or arms, but may manifest on any sun-exposed area [3]. When present on the scalp, they may be associated with non-scarring, patchy alopecia [4]. Histopathological findings in tumid lupus include a perivascular and periappendigal lymphocytic infiltrate without interface dermatitis and abundant interstitial mucin deposition [5]. Laboratory abnormalities, including reactive ANA, anti-Ro, anti-La, anti-dsDNA, and low 
complement may be present even in absence of concomitant systemic lupus erythematous [2]. Treatment with hydroxychloroquine is successful in most cases [2], although the associated alopetic lesions may require intralesional steroids [4]. For plaques without resolution with antimalarial treatment alone, addition of topical tacrolimus may be beneficial [6].

The differential diagnosis for tumid lupus includes pseudolymphoma and polymorphous light eruption. The clinical and histological picture of tumid lupus also has substantial overlap with Jessner's lymphocytic infiltration of the skin. However, some assert that tumid lupus more commonly occurs on the face and may show some evidence of basement membrane thickening [7]. Others view these conditions as a spectrum of disease rather than separate entities.

The cutaneous manifestations of hyper-IgM syndrome are usually consistent with signs of immunodeficiency, such as mucosal ulcers and warts [8]. Paradoxical autoimmune reactions, however, have been recognized in some patients [9]. In the AID-deficient variant, development of autoimmune disease, such as Crohn's disease, diabetes mellitus, autoimmune cytopenias and polyarthritis, has been reported [10]. Isolated cases of connective tissue disease, including systemic lupus erythematous and discoid lupus, in the setting of suspected autosomal recessive hyper-IgM syndrome have also been reported [11, 12]. In fact, a study conducted on patients with primary autoimmunity demonstrated that patients with higher levels of expression of surface IgM on B cells were at higher risk of development of autoimmunity [13]. While the mechanism for autoimmunity in this patient population has not been completely elucidated, a genetic basis has been suggested. Loss of function of the AID gene may lead to defects in self-tolerance, in addition to impaired somatic hypermutation and class switch recombination [14]. Thus, it is probable that mutations in immunoregulatory genes can lead to concomitant clinical manifestations of autoimmunity and immunodeficiency.

In conclusion, to our best knowledge, we report the first case of tumid lupus in a patient with a primary immunodeficiency syndrome. We emphasize the importance of clinicopathological correlation in arriving at a diagnosis, particularly when serological testing is unreliable or uninformative. Further, we highlight the phenomenon of paradoxical autoimmunity that can manifest as new cutaneous findings in an immunodeficient patient.

\section{Statement of Ethics}

The manuscript was prepared in compliance with all ethical and confidentiality guidelines and principles. Patient consent for biopsy and photography was obtained at the time of care.

\section{Disclosure Statement}

The authors have no conflicts of interest to disclose.

\section{Author Contributions}

Mirelys Barrios, Neera Nathan, Ryan Trowbridge, and Molly Plovanich contributed to the writing of the manuscript, as well as the editing and revising of the manuscript.

Dr. Rosalynn M. Nazarian contributed the histopathology images and their detailed interpretation applicable to the clinical context.

Dr. Daniela Kroshinsky is the senior author, overseeing and helping conceptualize and organize the main teaching points of the paper, writing as well as editing and revising the manuscript. 


\section{References}

1 Dekle CL, Mannes KD, Davis LS, Sangueza OP. Lupus tumidus. J Am Acad Dermatol. 1999 Aug;41(2 Pt 1):250-3.

2 Kuhn A, Richter-Hintz D, Oslislo C, Ruzicka T, Megahed M, Lehmann P. Lupus erythematosus tumidus-a neglected subset of cutaneous Lupus erythematosus: report of 40 cases. Arch Dermatol. 2000 Aug;136(8): 1033-41.

3 Rodriguez-Caruncho C, Bielsa I, Fernández-Figueras MT, Roca J, Carrascosa JM, Ferrándiz C. Lupus erythematosus tumidus: a clinical and histological study of 25 cases. Lupus. 2015 Jun;24(7):751-5.

4 Singh AH, Werth VP. Alopecia associated with papulonodular mucinosis (tumid) lesions of lupus erythematosus. J Clin Rheumatol. 1997 Dec;3(6):343-5.

5 Kuhn A, Sonntag M, Ruzicka T, Lehmann P, Megahed M. Histopathologic findings in lupus erythematosus tumidus: review of 80 patients. J Am Acad Dermatol. 2003 Jun;48(6):901-8.

6 Verma P, Sharma S, Yadav P, Namdeo C, Mahajan G. Tumid lupus erythematosus: an intriguing dermatopathological connotation treated successfully with topical tacrolimus and hydroxyxhloroquine combination. Indian J Dermatol. 2014 Mar;59(2):210.

7 Rémy-Leroux V, Léonard F, Lambert D, Wechsler J, Cribier B, Thomas P, et al. Comparison of histopathologicclinical characteristics of Jessner's lymphocytic infiltration of the skin and lupus erythematosus tumidus: multicenter study of 46 cases. J Am Acad Dermatol. 2008 Feb;58(2):217-23.

8 Chang MW, Romero R, Scholl PR, Paller AS. Mucocutaneous manifestations of the hyper-IgM immunodeficiency syndrome. J Am Acad Dermatol. 1998 Feb;38(2 Pt 1):191-6.

9 Jesus AA, Duarte AJ, Oliveira JB. Autoimmunity in hyper-IgM syndrome. J Clin Immunol. 2008 May;28(S1 Suppl 1):S62-6.

10 Quartier P, Bustamante J, Sanal O, Plebani A, Debré M, Deville A, et al. Clinical, immunologic and genetic analysis of 29 patients with autosomal recessive hyper-IgM syndrome due to Activation-Induced Cytidine Deaminase deficiency. Clin Immunol. 2004 Jan;110(1):22-9.

11 Arai J, Yasukawa M, Takada K, Tange Y, Saiki O, Horiuchi T, et al. Non-X-linked hyper-IgM syndrome with systemic lupus erythematosus. Clin Exp Rheumatol. 1998 Jan-Feb;16(1):84-6.

12 Wolpert KA, Webster AD, Whittaker SJ. Discoid lupus erythematosus associated with a primary immunodeficiency syndrome showing features of non-X-linked hyper-IgM syndrome. Br J Dermatol. 1998 Jun;138(6): 1053-7.

13 Picchianti Diamanti A, Rosado MM, Scarsella M, Ceccarelli S, Laganà B, D’Amelio R, et al. Increased serum IgM, immunodeficiency, and autoimmunity: A clinical series. Int J Immunopathol Pharmacol. 2015 Dec;28(4):54756.

14 Meyers G, Ng YS, Bannock JM, Lavoie A, Walter JE, Notarangelo LD, et al. Activation-induced cytidine deaminase (AID) is required for B-cell tolerance in humans. Proc Natl Acad Sci USA. 2011 Jul;108(28):11554-9. 